\title{
Effets Des Risques Climatiques Sur La Production Vivriere Dans La Commue De Lokossa (Benin)
}

\author{
Christian BAMAHOSSOVI \\ Franco-Néo S. C. DJESSONOU \\ Akibou A. AKINDELE
}

Département de Géographie et Aménagement du Territoire (Université

d'Abomey-Calavi)

Euloge OGOUWALE

Laboratoire Pierre PAGNEY, Climat, Eau, Ecosystème et Développement (LACEEDE)

doi: 10.19044/esj.2016.v12n29p370 URL:http://dx.doi.org/10.19044/esj.2016.v12n29p370

\begin{abstract}
The present study is a contribution to a better knowledge of the effects of the climatic risks on agriculture in the Commune of Lokossa.

The analysis of the climatological data (heights of rains and temperature) of the ASECNA (1973-2012) made it possible to determine the climatic risks. That of the agricultural data (1995-2012) made it possible to study the evolution of the agricultural outputs. Socio-anthropological information made it possible to apprehend the incidences of the climatic risks.

By the results obtained show that the floods (97\% of surveyed) and the drynesses ( $88 \%$ of surveyed) are the major climatic risks and recurring of the Commune of Lokossa. They appear through the delay in the starting of the rains, the insufficiency heights of rain due to the recurring ruptures of the precipitations recorded during rainy seasons as well as the lengthening of the dry seasons (99\% of surveyed). Supervening of the aforesaid risks generated floods of the fields and fading of the cultures ( $79 \%$ of surveyed) and caused during fifteen last years of the falls of the agricultural productions (15\% Zea mays, $20 \%$ Vigna unguiculata, $17 \%$ Arrachis hypogea).
\end{abstract}

Keywords: Township of Lokossa, risks climatic, vulnerability, strategies of adaptation, agriculture

Résumé

Les incidences des risques climatiques sur la production vivrière sont de plus en plus dommageables et récurrentes. La présente étude est une 
contribution à une meilleure connaissance des effets des risques climatiques sur l'agriculture dans la Commune de Lokossa.

L'analyse des données climatologiques (hauteurs de pluies et température) de l'ASECNA (1973-2012) a permis de déterminer les risques climatiques. Celle des données agricoles (1995-2012) a permis d'étudier l'évolution des rendements agricoles. Les informations socio-anthropologiques ont permis d'appréhender les incidences des risques climatiques.

Les inondations (97\% des enquêtés) et les sécheresses ( $88 \%$ des enquêtés) sont les risques climatiques majeurs et les plus récurrents dans la Commune de Lokossa. Ils se manifestent à travers le retard dans le démarrage des pluies, l'insuffisance des hauteurs de pluie due aux ruptures récurrentes des précipitations enregistrées au cours des saisons pluvieuses ainsi que l'allongement des saisons sèches (99 \% des enquêtés). Les survenances desdits risques ont engendré des inondations des champs et des flétrissements des cultures (79\% des enquêtés) et ont causé au cours des quinze dernières années des baisses des productions agricoles (15\% Zea mays, 20 \% Vigna unguiculata, 17 \% Arrachis hypogea).

Mots clés: Commune de Lokossa, risques climatiques, vulnérabilité, stratégies d'adaptation, agriculture

\section{Introduction}

Depuis les années 1970, l'Afrique de l'ouest est encline à la hausse de la température et à la récession pluviométrique (Bokonon-Ganta, 1987 et Adissoda, 2009). Ces dérèglements chroniques se caractérisent par une augmentation de la température moyenne, des sécheresses irrégulières et récurrentes, des perturbations des régimes pluviométriques. Les phénomènes qui découlent de la variabilité et du changement climatique engendrent des événements extrêmes qui ont frappé l'Afrique de l'Ouest ces dernières décennies (Amoussouga, 2011).

$\mathrm{Au}$ Bénin, la variation climatique se manifeste par une forte irrégularité des précipitations interannuelles tant dans leurs ampleurs que dans leurs répartitions (Boko, 1988). Ces variations climatiques sont la source des catastrophes multisectorielles qui accentuent la vulnérabilité des états et par ricochet celle des populations (IPCC, 2007).

Les pays en voie de développement comme le Bénin sont plus vulnérables que celles des pays riches (Banque Mondiale, 2008). En outre, de 2000 à 2004, les incidences induites par les catastrophes climatiques ont touché environ 262 millions de personnes dont $98 \%$ vivant dans des pays pauvres comme le Bénin (www.fao.org).

La figure 1 présente les situations géographique et administrative de la Commune de Lokossa. 


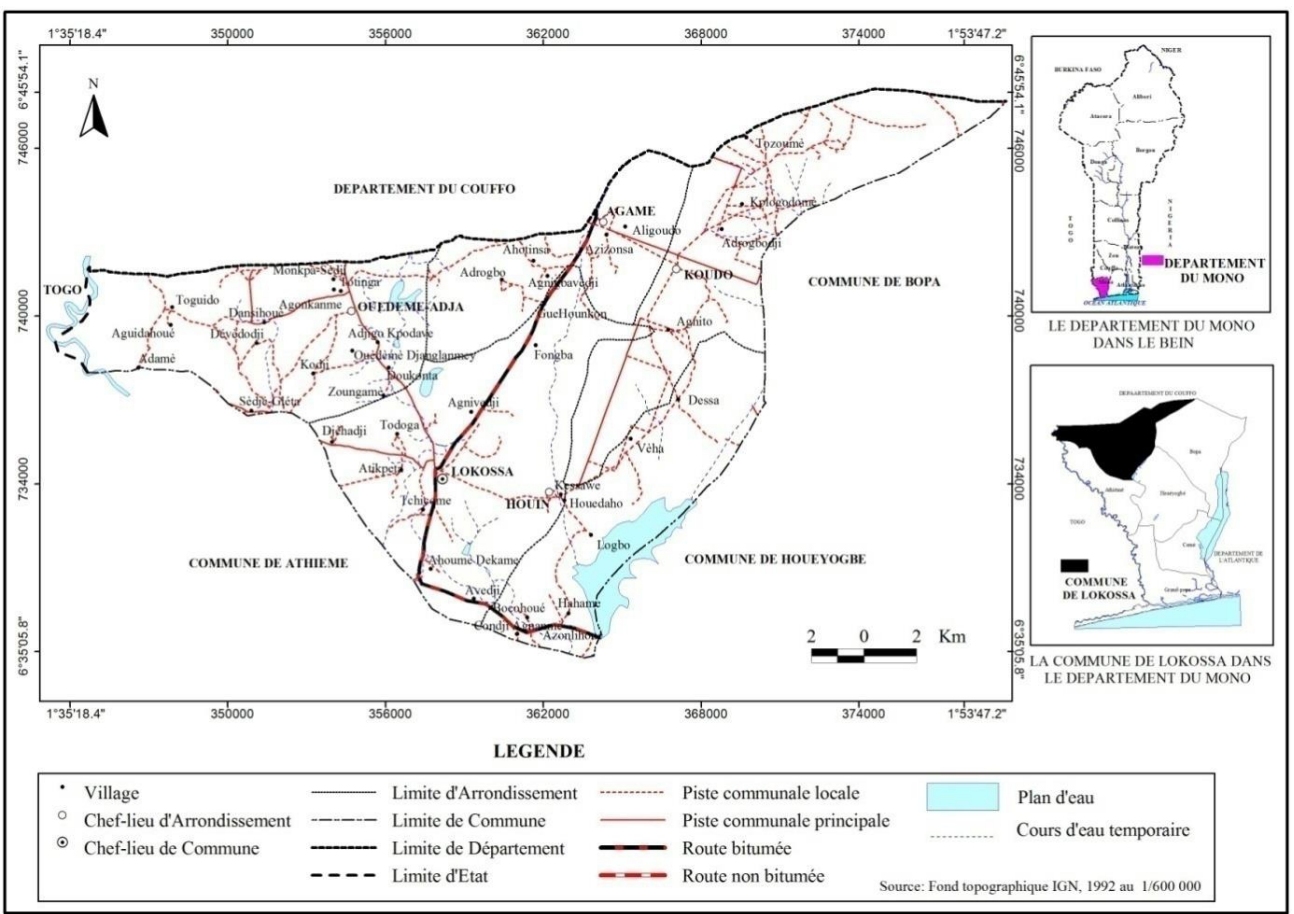

Figure 1 : Situations géographique et administrative de la Commune de Lokossa

La commune de Lokossa est située dans le département du Mono entre $6^{\circ} 34^{\prime} 52^{\prime}$ ' et $6^{\circ} 44^{\prime} 25^{\prime \prime}$ ' de latitude nord et entre $1^{\circ} 36^{\prime} 46^{\prime}$ ' et $1^{\circ} 52^{\prime}$ $17^{\prime \prime}$ de longitude est et couvre une superficie de $260 \mathrm{~km}^{2}$ pour une population de 109839 habitants repartie dans cinq arrondissements.

\section{Matériels et méthodes}

Les données climatologiques (hauteurs de pluies et températures) de 1953 à 2012 de l'ASECNA ont permis d'analyser la dynamique des paramètres climatiques. Les statistiques agricoles du CARDER Mono/ Couffo de 1995 à 2012 ont servie à déterminer l'évolution des cultures principales de la Commune. Un appareil photo a permis de prendre des vues de crises agricoles.

Les cinq arrondissements de la Commune de Lokossa ont été investigués et les personnes interrogées répondent aux critères :

$>$ être chef de ménage agricole, âgé d'au moins cinquante ans et résidant dans l'un des cinq arrondissements pour être capable de renseigner sur les catastrophes agricoles induites par les risques climatiques ;

avoir pratiqué les activités agricoles au cours des vingt dernières années dans la Commune de Lokossa pour fournir des informations agricoles fiables sur la Commune. 
La taille de l'échantillon au niveau de chaque arrondissement est déterminée suivant la formule de Schwartz (1995) et qui s'écrit : $\mathrm{x}=\mathrm{Z} \alpha 2 \mathrm{x} \mathrm{pq} / \mathrm{i} 2$ avec $\mathrm{x}$ $=$ taille de l'échantillon, $Z \alpha=1,96$ écart réduit correspondant à un risque $\alpha$ de $5 \% ; \mathrm{p}=\mathrm{n} / \mathrm{N}$ avec $\mathrm{p}=$ proportion des ménages de chaque arrondissement (n) par rapport au nombre de ménages agricoles de la Commune $(\mathrm{N})$ dans laquelle se situe ce dernier; $q=1-\mathrm{p}$; et $\mathrm{i}=5 \%$.

Questionnaires, grilles d'observation et guides d'entretien sont les matériels utilisés techniquement à travers les observations directes, les entretiens directs et la Méthode Active de Recherche Participative (MARP) pour la collecte d'informations relatives au incidences des risques climatiques sur la production agricole et les stratégies d'adaptation développées. Les données collectées ont été traitées manuellement et statistiquement à l'aide des logiciels Excel 2007 pour la réalisation des graphiques et le logiciel Arc View 3.2 pour la réalisation de cartes.

\section{Résultats et discussion}

\subsection{Indicateurs de risques climatiques}

Les risques climatiques sont causés dans la Commune de Lokossa par la variabilité des paramètres climatiques tels que les hauteurs de pluies et les températures. La figure 2 présente l'évolution des hauteurs pluviométriques enregistrées au cours de la période allant de 1973 à 2012.

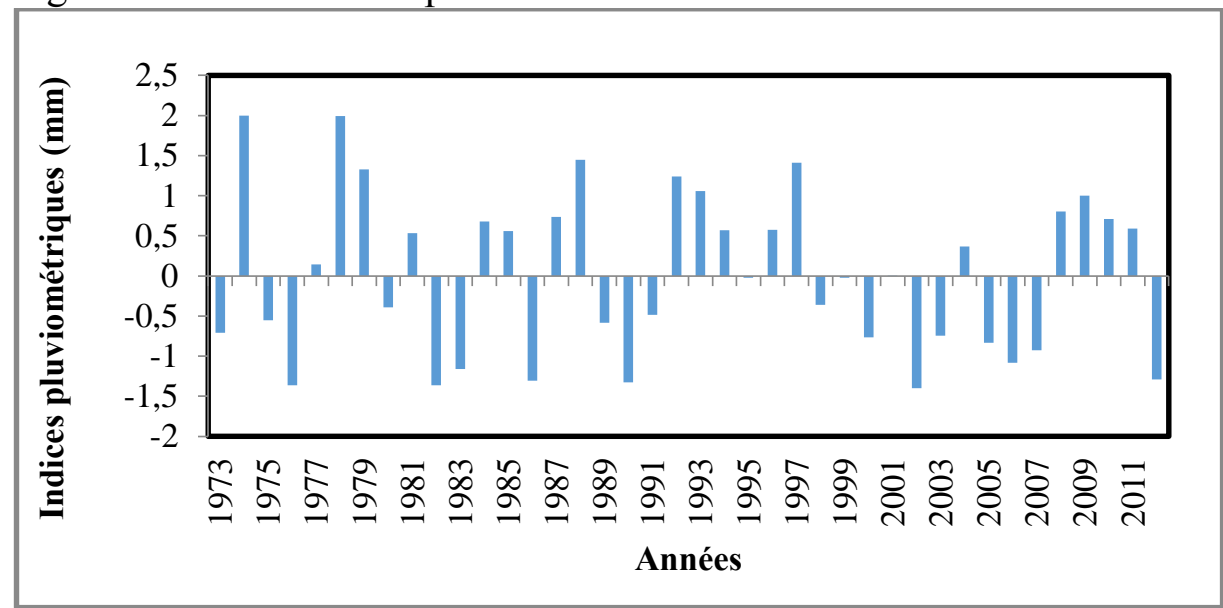

Figure 2 : Indices pluviométriques de la période allant de 1973 à 2012 Source : ASECNA, 2013

L'analyse de la figure 2 montre qu'il a été enregistré au cours de la période allant de 1973 à 2012, 35 \% d'années extrêmement excédentaires, $25 \%$ d'années normales et $40 \%$ d'années extrêmement déficitaires. En outre, les années excédentaires et les années déficitaires sont des périodes au cours 
desquelles respectivement les excès et les insuffisances de la pluviosité ont été très préjudiciables pour les cultures dans la Commune de Lokossa.

L'évolution des hauteurs de pluies journalières en 24 heures est présentée à la figure 3 .

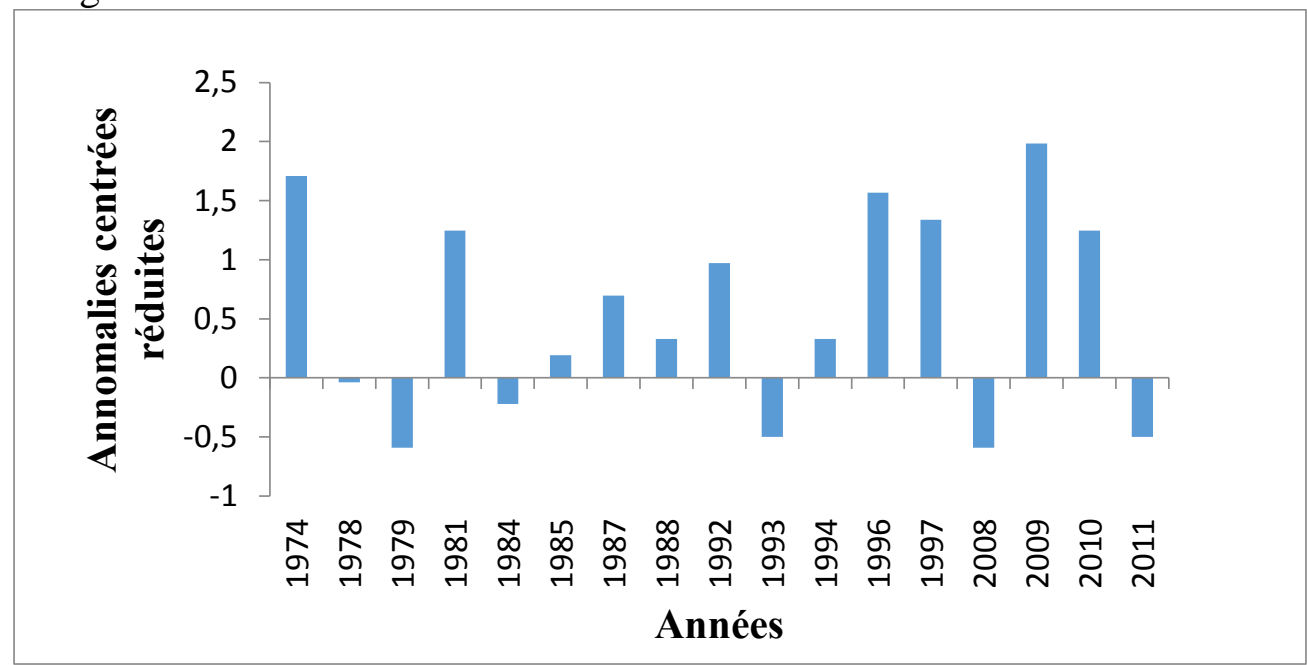

Figure 3 : Variabilité interannuelle des hauteurs de pluies maximales en 24 heures Source de données : ASECNA, 2013

L'examen de la figure 3 révèle que la Commune de Lokossa a connue au cours de la période allant de 1973 à 2012 onze années d'inondations dont les plus importantes ont été celles de 1974, 1996 et 2009. En effet, 57 \% des personnes interrogées ont confirmé que ces années ont effectivement des années d'inondations au cours desquelles elles ont enregistré de grosses pertes agricoles. Outre les variations pluviométriques, il a été aussi enregistré des fluctuations thermométriques (figure 4).

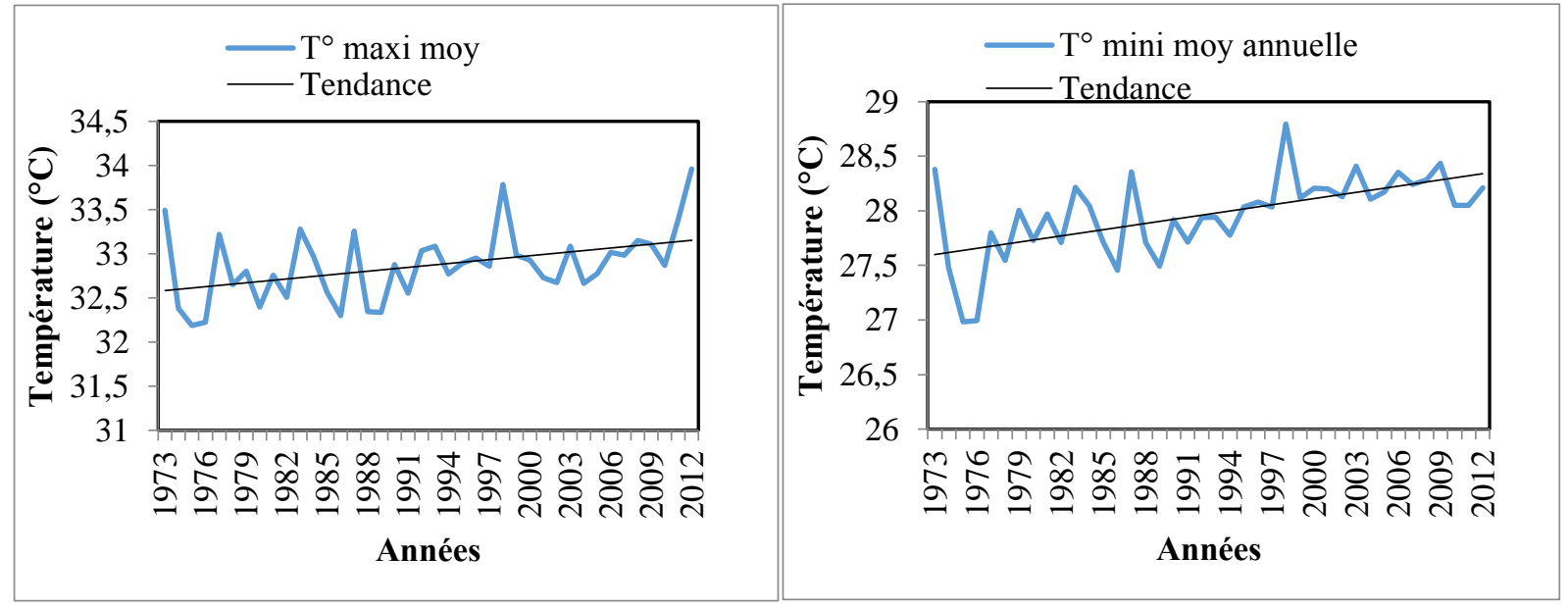

Figure 4 : Variation interannuelle des températures au cours de la période allant de 1973 à 2012

Source de données : ASECNA, 2013 
L'analyse de la figure 4 fait constatée une tendance à la hausse des températures maximales et minimales moyennes au cours de la période allant de 1973 à 2012. En effet, elles ont connu des hausses respectives de $1,78^{\circ} \mathrm{C}$ et de $1,82{ }^{\circ} \mathrm{C}$ au cours de ladite série. Cette hausse spectaculaire des températures est à la base des canicules qui freinent la bonne exécution des activités des populations et surtout des travaux champêtres à cause des troubles tels que les vertiges, les évanouissements et les fourmillements qu'elles causent (Bamahossovi, 2014).

\subsection{Incidences des risques climatiques sur les principales cultures}

Les cultures retenues pour cette étude sont le maïs (Zea mays), l'arachide (Arrachis hypogea) et le niébé (Vigna unguiculata) du fait de leurs importances dans l'alimentation des populations de Lokossa. La figure 5 présente l'évolution desdites cultures au cours de la période allant 1995 à 2012.

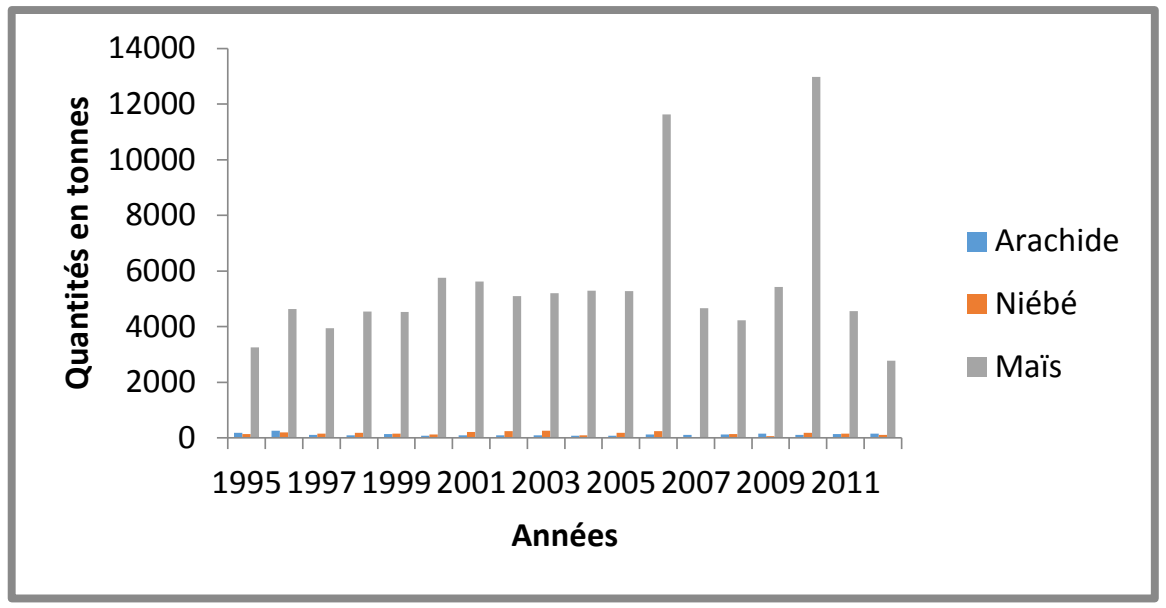

Figure 5: Evolution des cultures principales de 1995 à 2012

Source : MAEP, 2013

La figure 5 fait état des pertes induites par les risques climatiques au cours de la période allant de 1995 à 2012. En effet, il a été enregistré au cours de ladite période une réduction de $17 \%$, de $20 \%$ et de $15 \%$ respectivement des productions d'arachide (Arrachis hypogea), de niébé (Vigna unguiculata) et de maïs (Zea mays). La planche 1 présente respectivement les incidences des inondations et des sécheresses sur les cultures. 

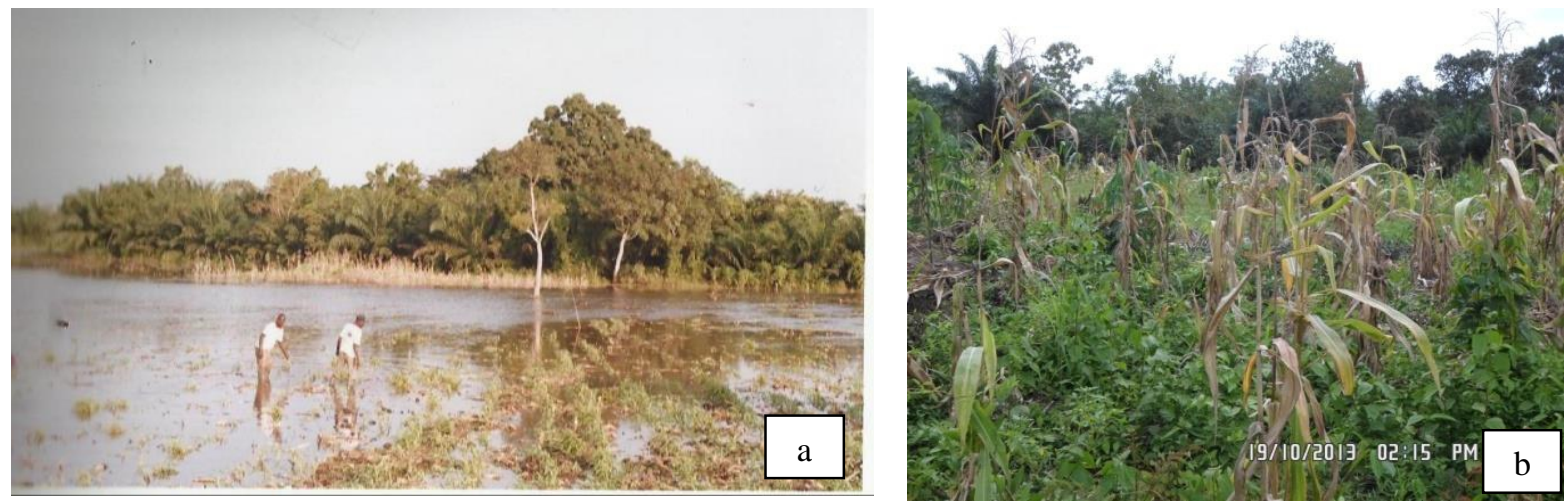

Planche 1 : Incidences des inondations et des sécheresses sur les cultures Prise de vues : Montin 2012 et Bamahossovi 2013

L'observation de la planche 1 fait constater que les excès (a) et les déficits (b) pluviométriques causent des pertes agricoles aux paysans de la Commune de Lokossa du fait de la dépendance de l'agriculture dans cette Commune des paramètres climatiques. Les inondations et les sécheresses ont été respectivement pour $97 \%$ et $88 \%$ des paysans interrogés les plus catastrophiques au cours des deux dernières décennies. Elles se sont manifestées à travers le retard dans le démarrage des pluies, l'insuffisance des hauteurs de pluie due aux ruptures récurrentes des précipitations enregistrées au cours des saisons pluvieuses ainsi que l'allongement des saisons sèches ( $99 \%$ des paysans interrogés).

Cette situation rend vulnérables les paysans qui n'arrivent plus à faire face à leurs besoins socio-économiques (Akindélé, 2013). Face à ces situations, les paysans de la Commune de Lokossa ont développé des stratégies qui ont montré leurs limites.

\subsection{Stratégies d'adaptation paysannes aux risques climatiques}

Les inondations et les sécheresses ont été les risques les plus préjudiciables induits par les variabilités climatiques aux producteurs agricoles dans la Commune de Lokossa (99 \% des enquêtés) et auxquels ils ont développé des stratégies de survie. La planche 2 est une illustration des stratégies d'adaptation développées par les paysans de la Commune de Lokossa. 

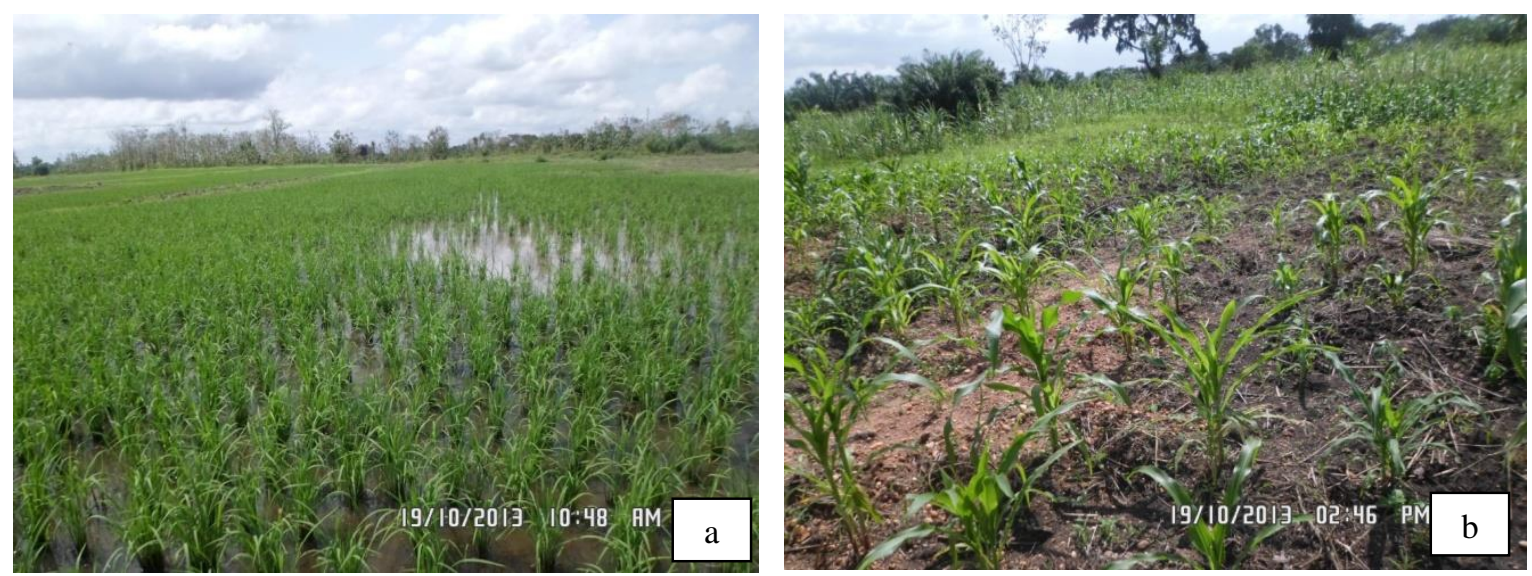

Planche 2 : Stratégies d'adaptation développées par les paysans

Prise de vues : Bamahossovi, 2013

La planche 2 témoigne de la mise en valeur rizicole de zones marécageuses (a) et de l'adoption des semis échelonnés (b) comme stratégies d'adaptation développées respectivement par $58 \%$ et $67 \%$ des paysans de la Commune de Lokossa. Ces résultats confirment ceux de de Tassou (2013), Houssou-Goé (2008) et de Ogouwalé (2006) selon lesquels les semis échelonnés et la culture des bas-fonds sont des stratégies paysannes aux catastrophes agricoles. Le tableau I présente la synthèse des stratégies développées et leurs contraintes pour les paysans.

Tableau I : Synthèse des stratégies d'adaptation et des contraintes

\begin{tabular}{|l|l|}
\hline Mise en valeur des bas-fonds & Défrichage difficile \\
\cline { 2 - 2 } & Risques d'inondation \\
\cline { 2 - 2 } & Non maîtrise des techniques d'exploitation \\
\cline { 2 - 2 } & Risques pathologiques \\
\cline { 2 - 2 } & Développement rapide des adventices \\
\hline Semis échelonnés & Impossibilité de récolter d'un coup (récoltes multiples) \\
\cline { 2 - 2 } & Temps supplémentaire alloué pour les resemis \\
\cline { 2 - 2 } & Demande plus d'effort \\
\hline
\end{tabular}

Source : Akindélé, 2013 et Bamahossovi, 2014

L'observation du tableau I montre l'inefficience des stratégies d'adaptation développées par les paysans de la Commune de Lokossa. A cet effet, il est impérieux d'intensifier le reboisement pour accroitre la séquestration du carbone (Sounou, 2013) et de promouvoir l'usage du gaz domestique afin limiter l'action destructrice des gaz à effet de serre sur la couche d'ozone.

\section{Conclusion}

Dans la Commune de Lokossa, les inondations et les sécheresses sont les risques climatiques qui ont été les plus catastrophiques pour les paysans du fait de la dépendance de leur activité de revenue qu'est l'agriculture des 
paramètres climatiques. En effet, ils ont causé au cours de la période allant de 1995 à 2012 des réductions de l'ordre de $15 \%$, de $20 \%$ et de $17 \%$ respectivement des cultures telles que le maïs (Zea mays), le niébé (Vigna unguiculata) et l'arachide (Arrachis hypogea). Face à cette situation, $58 \%$ et $67 \%$ des paysans de la Commune de Lokossa ont procédé respectivement à la mise en valeur agricole des bas- fonds et à l'adoption des semis échelonnés qui leurs sont très contraignantes.

\section{References:}

1. Adissoda O. K. (2009) : Vulnérabilité des populations au paludisme et aux Infections Respiratoire Aiguës (IRA) dans le contexte des changements climatiques : cas de la Commune de Parakou. Mémoire de maîtrise de géographie. DGAT/FLASH/UAC, $82 \mathrm{p}$.

2. Akindélé A. A. (2013) : Vulnérabilité et adaptation de la production vivrière aux contraintes climatiques dans la Commune de AdjaOuèrè. In AIC, Cotonou, $6 \mathrm{p}$.

3. Amoussouga E. B. (2011): Activités humaines par épisodes climatiques annuel et effort d'adaptation des populations sur le plateau d'Abomey. Mémoire de maîtrise de géographie. DGAT/FLASH/UAC ,77 p.

4. Bamahossovi C. (2014) : Gestion des catastrophes climatiques dans la Commune de Lokssa. Mémoire de maîtrise en géographie, DGAT/ FLASH/ UAC, 76 p.

5. Banque Mondiale (2008): L'agriculture au service du développement. Rapport sur le développement dans le monde, Washington, $28 \mathrm{p}$.

6. Boko M. (1988) : Climats et communautés rurales du Bénin : Rythmes climatiques et rythmes de développement. Thèse de doctorat d'Etat ès Lettres et Sciences Humaines. CRC, URA 909 du CNRS, Université de Bourgogne, Dijon, 2 volumes, 608 p.

7. Bokonon-Ganta E. B., 1987 : Les climats de la région du Golfe du Bénin. (Afrique Occidentale). Thèse de doctorat du 3ème cycle, Paris IV, Sorbonne, 248p + Annexes.

8. Houssou-Goé S. S. P. (2008) : Agriculture et changements climatiques au Bénin : Risques climatiques, vulnérabilité et stratégies d'adaptation des populations rurales du département du Couffo. Thèse pour l'obtention du diplôme d'ingénieur agronome. DESAC/ FSA/ UAC, 140 p.

9. INSAE (2011) : Inondation au Bénin: Rapport d'évaluation des besoins post catastrophes. Cotonou, $82 \mathrm{p}$. 
10. IPCC (2007): Observed changes on climate and their effects. Fourth assessment report. Synthesis report, $21 \mathrm{p}$.

11. Montin G. (2012) : Effet des crues sur la production agricole dans l'arrondissement de Ouèdèmè-Adja (Commune de Lokossa). Mémoire de maîtrise de géographie. DGAT/FLASH/UAC, $86 \mathrm{p}$.

12. Ogouwalé E. (2006) : Changements climatiques dans le Bénin méridional et central : indicateurs, scénarios et prospectives de la sécurité alimentaire. Thèse de Doctorat Unique, EDP/FLASH, UAC, 302 p.

13. Sounou (2013) : Etude comparative de la capacité de séquestration du carbone de Tectona grandis et de Gmelina arborea. Mémoire de master. RESBIO/ FSA/ UAC, $57 \mathrm{p}$.

14. Tassou M. (2013) : Variabilité climatique et production agricole dans la Commune de Bassila. Mémoire de maîtrise, DGAT/ FLASH/ UAC, 88 p.

15. www.fao.org consulté le 27 Août 2013 à 18 h 35 mn 